\title{
Development of Sleep Support System Using Electroencephalogram for Person with Developmental Disorders
}

\author{
Sakiko Ogoshi, ${ }^{1 *}$ Yasuhiro Ogoshi, ${ }^{2}$ Toru Saitou, ${ }^{1}$ \\ Hitoshi Nishi, ${ }^{1}$ Yoshinori Mitsuhashi, ${ }^{3 \dagger}$ and Akio Nakai ${ }^{4 \dagger \dagger}$ \\ ${ }^{1}$ Department of Electrical Engineering and Computer Science, \\ National Institute of Technology, Fukui College, Geshi-cho, Sabae-shi, Fukui 916-8507, Japan \\ ${ }^{2}$ Graduate School of Engineering, University of Fukui, 3-9-1 Bunkyo, Fukui 910-8507, Japan \\ ${ }^{3}$ Faculty of Education and Regional Studies, University of Fukui, 3-9-1 Bunkyo, Fukui 910-8507, Japan \\ ${ }^{4}$ Hyogo Children's Sleep and Development Medical Research Center, \\ Akebono-cho 1070, Nishi-ku, Kobe 651-2181, Japan
}

(Received January 15, 2018; accepted June 13, 2018)

Keywords: developmental disorders, sleep support system, EEG

In recent years, the number of children requiring special support has increased significantly, and satisfying the support and educational needs of such individuals has become a critical concern. Many students have been diagnosed with one or more developmental disorders, such as Asperger syndrome, high functioning autism, attention deficit disorder, hyperactivity disorder, and learning disabilities. Some special-needs children are attention deficit hyperactivity disorder (ADHD) hyperactive/impulsive and exhibit certain behaviors such as excessive fidgeting, talking out of turn, and running around. Other special-needs children are ADHD inattentive, who are often distracted and forget things at home or school. Such children also experience difficulties during organized activities. These difficulties can have a negative impact on a child's learning and self-confidence. Addressing such difficulties as early as possible can have a positive impact on the children's performance in school. In this study, we propose a collaborative system that can be utilized by teachers, parents, and supporters. Individual support equipment using Internet of Things (IoT) and sensors is necessary. Support observation of sleep state and support for sleep onset are also necessary. We therefore proposed and developed a sleep support system.

\section{Introduction}

In recent years, the number of students who struggle in school has increased in Japan. Many such students have been diagnosed with one or more developmental disorders, such as autistic spectrum disorder (ASD), attention deficit hyperactivity disorder (ADHD), and learning

\footnotetext{
*Corresponding author: e-mail: sakiko_ogoshi@yahoo.co.jp

${ }^{\dagger}$ Current address: Faculty of Education, Humanities and Social Sciences, Division of Teacher Education, Department of Science of Human Development, Psychology of Handicapped Child

${ }^{\dagger}$ Current adress: Institute for Education, Mukogawa Women’s University, 6-46 Ikebiraki, Nishinomiya, 663-8558, Japan http://dx.doi.org/10.18494/SAM.2018.1908
} 
disorders (LD). Children with these disorders can receive inadequate support, and the impact of these disorders is serious. ${ }^{(1-10)}$ Therefore, we must support persons with developmental disorders individually because each person has individual needs and disorder characteristics. Developmental disorders have been recently estimated to afflict as much as about $6.5 \%$ of students in regular classes. ${ }^{(1)}$ In 2012, a survey was conducted by the Japan Ministry of Education, Culture, Sports, Science and Technology of elementary and junior high school students across the country. Of the 52272 cases that the surveyed schools reported, an estimated 6.5 percent of children attending regular classes had significant learning or behavioral problems, indicating that two students per class might have a suspected developmental disorder. For children with developmental disorders, special support is required to aid them in many aspects of life, and individual education support is of critical importance.

Recent developments in information and communication technology (ICT) for special education have been significant. Recent studies have examined the benefits of various forms of ICT tools for children with developmental disorders. ${ }^{(6)}$ There has been increasing interest in assessing children with special needs using ICT systems to overcome difficulties in the learning process. ICT can maintain a child's skills and build an appropriate learning environment relative to their individual needs and curriculum requirements. ICT in special education can provide children with numerous opportunities for a rich learning experience relevant to their growth and positive outcomes with respect to their learning difficulties.

Children spend most of their time in school with teachers or at home with their parents; thus, it would be beneficial if teachers and parents were able to assess and react to specific behavioral challenges. ${ }^{(7)}$ Therefore, we developed the ICT support system for individual education that parents, teachers, and supporters can use to share information. ${ }^{(12,13)}$ This support system proposes and provides individuals with support on the basis of their behavior characteristics. Among the children with developmental disorders, some have problems in sleeping at night, and consequently they, for example, fall asleep during class.

Previous studies showed that some children with developmental disorders have sleep problems. The negative associations with childhood sleep problems are of particular concern in view of the high prevalence of sleep difficulties. Figures of about $25 \%$ of preschool children, $43 \%$ of school-age prepubescent children, and 33\% of adolescents have been given. The reported rates of sleep disorders in children with developmental disorders are even higher. Rates vary depending on the criteria used to define a 'sleep problem' but examples reported are $49-89 \%$ of children with ASD, $25-50 \%$ of children with ADHD, and 34-86\% of children with intellectual disabilities. ${ }^{(14)}$ Thus, we developed a sleep support system, and we need to measure the quality of sleep for the development of the sleep support system. Therefore, in this research, to develop a device that supports sleep, we aimed to develop an index that measures the quality of sleep in a short time and proposed a system that performs biofeedback that supports sleep when sleep is difficult. 


\section{Proposal and Development of Sleep Support System}

As a result of an interview survey on a person who has difficulty in falling asleep, we propose and develop sleep support equipment that changes the temperature of the pillow or bed to induce sleep by warming up the bed in winter and cooling it in summer when it is difficult to sleep.

We developed a sleep support system that parents and supporters can use to share information. The collaborative support system for developmental disorders harnesses a support system using IoT support equipment and sensors. Figure 1 shows the outline of the sleep support system. Figure 2 shows the structure of the sleep support system.

\section{Development of Short-time Sleep Quality Index Using Electroencephalogram (EEG)}

To use the sleep support system, it is necessary to measure the quality of sleep because there is no need to use the sleep support system when the sleep quality is good. As a method,

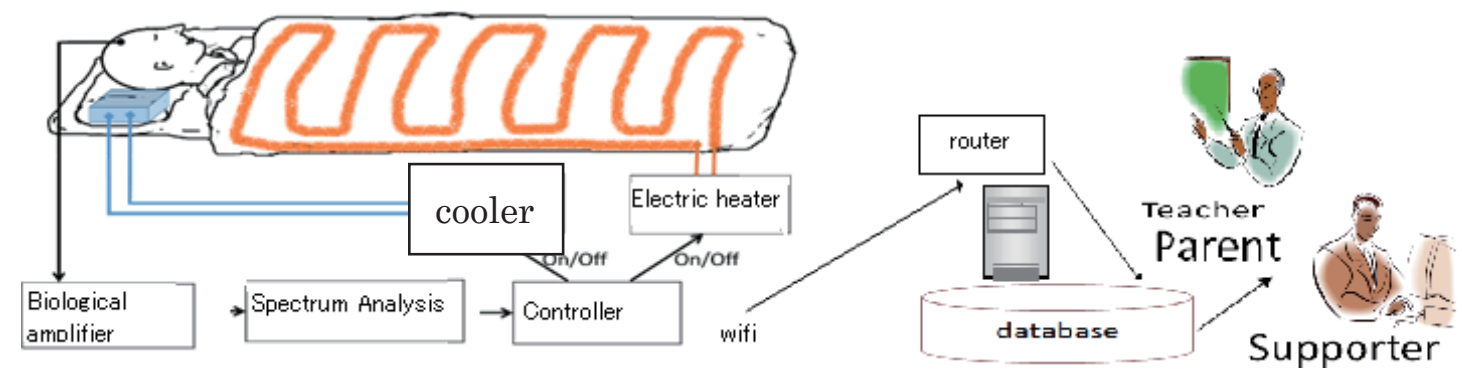

Fig. 1. (Color online) Outline of sleep support system.

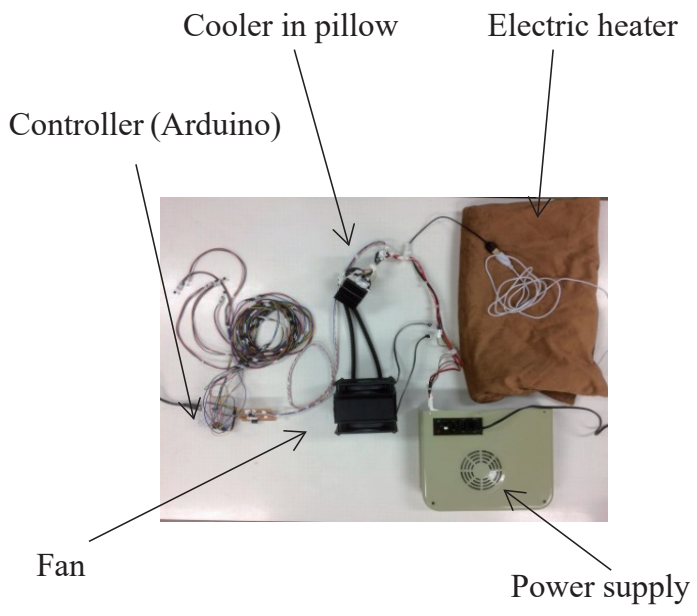

Fig. 2. (Color online) Structure of sleep support system. 
we developed an index to measure the sleep quality and investigated it. Electrodes can be easily removed, which is a problem when measuring brain waves during sleep. Moreover, it is difficult to sleep if cap-type electrodes are used. In non-rapid eye movement (non-REM) sleep, stages such as 1, 2, 3, and 4 are often measured. However, this method takes a long time and it is easy to take out the electrode, so it is desirable to develop an index that can be checked in 5 min so that we can determine the sleep quality as soon as a person starts sleeping. Since it is easy to take out the electrode at the back of the head, we decided to use the top of the head. The purpose of this study is to investigate the 5-min sleep quality after the start of sleep by EEG analysis and check if it can indicate subjective sleepiness. We observed a decrease in the spectrum of the alpha wave band related to the quality of induction of natural sleep, and examined whether it matches the subjectivity of easy sleep.

\subsection{Participants}

There were nine male and female participants (age: 18 to 20 years old).

\subsection{Recoding}

Data were obtained from two electrodes across the scalp. Data from the electrode sites C3 and $\mathrm{C} 4$ are presented (Fig. 3). The data were taken from the power spectrum of the alpha wave $(8-13 \mathrm{~Hz})$ for each period.

\subsection{Task condition}

The subjects took a 5-min nap. After the EEG session, the subjects had to say whether falling asleep was easy or difficult.

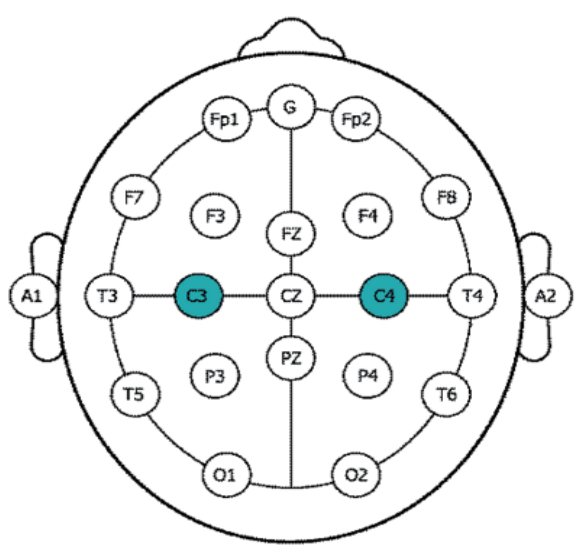

Fig. 3. (Color online) 10-20 method of electrode placement. 


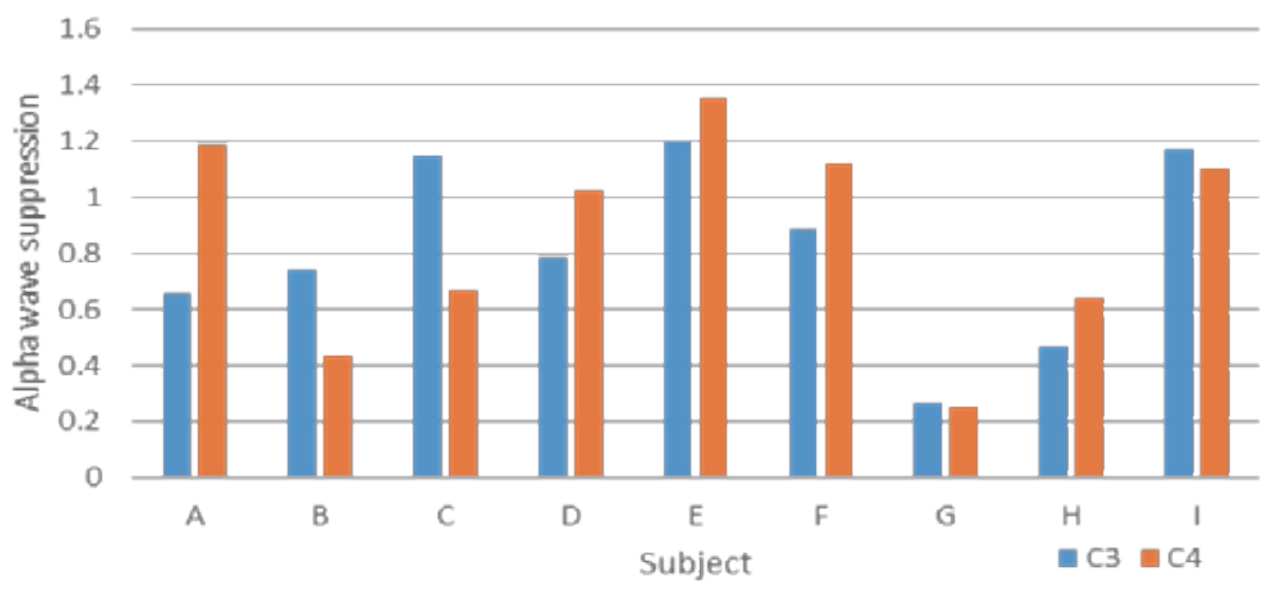

Fig. 4. (Color online) Values of individual alpha wave suppression.

\subsection{Sleep EEG analysis}

The sampling rate was $500 \mathrm{~Hz}$ EEG and the band pass filter was $0.1-30 \mathrm{~Hz}$. The integrated power in the $8-13 \mathrm{~Hz}$ range was computed using a Fast Fourier Transform. Data were segmented into epochs of $2 \mathrm{~s}$ beginning at the start of the segment. Fast Fourier Transforms were performed on the epoched data (1024 points). Alpha wave suppression was calculated by determining the ratio of the power under the experimental conditions to that under the baseline condition.

[Alpha wave suppression $]=[$ integrated power in the $8-13 \mathrm{~Hz}$ range $(0-120 \mathrm{~s})] /[$ integrated power in the $8-13 \mathrm{~Hz}$ range of $(160-280 \mathrm{~s})]$.

\subsection{Result of sleep EEG analysis}

Figure 4 shows values of individual alpha wave suppression. The results of a subjective questionnaire showed that Subjects C, E, and I answered "Falling asleep was difficult". In the $\mathrm{C} 3$ position of Subjects $\mathrm{C}, \mathrm{E}$, and I, alpha wave suppression did not occur. The $\mathrm{C} 3$ alpha wave was reduced when falling asleep was easy. Thus, it can be considered that if the alpha waves are not suppressed, biofeedback is useful.

\section{Conclusion}

We proposed a sleep support system using EEG. While this system supports sleeping, it can share information on sleeping state with parents and supporters. We showed the possibility of using the sleep support system by a simple method using alpha waves. As a future task, we will continuously verify the effectiveness of indicators and propose biofeedback methods individually. We will also examine the relationship between sleep data and daytime behavior data. 


\section{Acknowledgments}

This work was supported by KAKENHI 15K04553 and 15K01752 and SCOPE (162305008). This research has obtained ethics review approval.

\section{References}

1 I. Hertz-Picciotto and L. Delwiche: Epidemiology 20 (2009) 84.

2 G. Polanczyk, M. S. de Lima, B. L. Horta, J. Biederman, and L. A. Rohde: Am. J. Psychiatry 164 (2007) 942.

3 M. Altarac and E. Saroha: Pediatrics 119 (2007) 77.

4 G. D. Tsiotra, A. D. Flouris, Y. Koutedakis, B. E. Faught, A. M. Lane, and N. Skenteris: J. Adolesc. Health 39 (2006) 125.

5 É.Slattery, J. McMahon, and S. Gallagher: Res. Dev. Disabilities 65 (2017) 12.

6 C. Xu, R. Reid, and A. Steckelberg: Educ. Treat. Children 25 (2002) 224.

7 S. D. Athanasios and E. I. Rodi: Int. J. Emerging Technol. Learn. 8 (2013) 41.

8 R. A. Barkley: J. Clin. Psychiatry, Educ. Treat. Children 25 (2002) 224.

9 G. Cervellin and G. Lippi: Eur. J. Internal Med. 22 (2011) 371.

10 R. Koyanagi, K. Noboru, and K. Natsume: IEICE Tech. Rep. 113 (2013) 21.

11 http://www.mext.go.jp/a menu/shotou/tokubetu/material/ icsFiles/afieldfile/2012/12/10/1328729 01.pdf

12 S. Ogoshi and Y. Ogoshi: Med. Sci. Digest 43 (2017) 487 (in Japanese).

13 T. Saitoh, S. Ogoshi, Y. Takaku, and S. Ishigami: J. Soc. Plant Eng. Jpn. 29 (2017) 13 (in Japanese).

14 L. Wiggs: J. R. Soc. Med. 94 (2001) 177. https://www.ncbi.nlm.nih.gov/pmc/articles/PMC1281388/ 